\title{
Characteristics of Cognitive Impairment in Patients With Post-stroke Aphasia
}

\author{
Boram Lee, MD, Sung-Bom Pyun, MD, PhD
}

\author{
Department of Physical Medicine and Rehabilitation, Korea University College of Medicine, Seoul, Korea
}

\begin{abstract}
Objective To analyze cognitive functions of post-stroke aphasia patients compared to patients having right hemispheric stroke and left hemispheric lesions without aphasia, and to look for a relationship between cognitive deficits and aphasia severity.

Methods Thirty-six patients with right hemispheric stroke (group 1), 32 with left hemispheric lesion without aphasia (group 2), and 26 left hemispheric stroke patients with aphasia (group 3) completed a set of tests in the computerized neurocognitive function batteries for attention, executive function and intelligence and Korean version of Western Aphasia Battery. Data analyses explored cognitive characteristics among the three groups and the correlation between cognitive deficits and aphasia severity.

Results Right hemispheric and left hemispheric stroke patients without aphasia showed similar findings except for digit span forward test. Cognitive tests for working memory and sustained attention were significantly impaired in the aphasic patients, but intelligence was shown to be similar in the three groups. Significant correlation between cognitive deficit and aphasia severity was only shown in some attention tests.

Conclusion Cognitive deficits may be accompanied with post-stroke aphasia and there are possible associations between language and cognitive measures. Therefore, detection and treatment towards coexisting cognitive impairment may be necessary for efficient aphasia treatment.
\end{abstract}

Keywords Stroke, Aphasia, Cognition

\section{INTRODUCTION}

Aphasia and cognitive impairment are common consequences following stroke. It is well-known that indi-

\footnotetext{
Received December 2, 2013; Accepted July 16, 2014

Corresponding author: Sung-Bom Pyun

Department of Physical Medicine and Rehabilitation, Korea University Anam Hospital, 73 Inchon-ro, Seongbuk-gu, Seoul 136-705, Korea Tel: +82-2-920-6471, Fax: +82-2-929-9951, E-mail: rmpyun@korea.ac.kr

(c) This is an open-access article distributed under the terms of the Creative Commons Attribution Non-Commercial License (http://creativecommons. org/licenses/by-nc/3.0) which permits unrestricted noncommercial use, distribution, and reproduction in any medium, provided the original work is properly cited.

Copyright ( 2014 by Korean Academy of Rehabilitation Medicine
}

viduals with right hemispheric strokes tend to have some degree of deficit in attention and visual-spatial recognition, but less amount of literature are present regarding the nonlinguistic cognitive deficits of patients with left hemisphere stroke. There has been some evidence that nonlinguistic cognitive deficits, such as attention, working memory, and executive functions, and aphasia, frequently coexist in the left hemispheric stroke patients [1-5].

The less amount of literature may be partly due to the difficulty of assessing nonverbal cognitive functions in the presence of aphasia and stroke, which is well-known [6-9]. There can be communication limitations in the assessment, or some tests just may be inappropriate for 
aphasic patients, like the Stroop test, for an example, where rapid naming and reading skills are required. Since most of the patients with left hemispheric strokes are right handed, motor deficits can also impede patient performance.

The concern to the concurrent cognitive impairment in patients with post-stroke aphasia is that impaired learning ability may limit rehabilitation efficacy, including language therapy in patients with aphasia $[10,11]$. Therefore, the detection and following treatment of nonverbal cognitive impairment come down to an important portion in the rehabilitation of a patient with post-stroke aphasia.

This study has mainly two purposes 1) to analyze the patterns of cognitive deficits in patients with post-stroke aphasia, 2) to look for a relationship between cognitive deficits and aphasia severity. Additionally, we aimed to find out which domain of language skill, if any, may be particularly responsible for the correlation.

\section{MATERIALS AND METHODS}

\section{Subjects}

Ninety-four patients with radiologically confirmed unilateral right or left hemisphere stroke who were admitted and evaluated within 3 months after onset in the Department of Physical Medicine and Rehabilitation of the Korea University Medical Center between May 5, 2007 and July 20, 2012, were recruited retrospectively. All participants were right-handed and native Korean speakers. Their lesions were confirmed by magnetic resonance imaging or computed tomography. The study included subjects older than 21 years of age. Exclusion criteria were lesions involving bilateral hemisphere, concurrent conditions and disorders that might affect any cognitive skills under study, traumatic brain injury, infratentorial lesions, or patients with right hemispheric lesions who were assessed to have aphasia (crossed aphasia) by a speech language pathologist (SLP).

Ninety-four patients were divided to 3 groups based on their laterality of lesion and the result of language evaluation. Group 1 included patients with right hemispheric stroke ( $n=36)$. Group 2 consisted of those with left hemispheric lesion but no aphasia $(\mathrm{n}=32)$. Group 3 comprised left hemispheric stroke patients with aphasia $(n=26)$.

\section{Tests and measurements}

General characteristics including biographical and neurologic data were collected by chart review. For the assessment of cognitive function, Computerized Neurocognitive Test (CNT; MaxMedica, Seoul, Korea) was performed for every patient. The following nine subtests were included: digit span test forward (DST-F) and backward (DST-B), visual span test forward (VST-F) and backward (VST-B), visual and auditory continuous performance test (VCPT and ACPT), Trail Making Test (TMT), Wisconsin card sorting test (WCST), and Raven colored progressive matrices (RCPM). DSTs involved listening to certain amount of digits and repeating them after a moment in order for DST-F, and backwards for DST-B. Verbal attention and memory were tested with DSTs, and especially with DST-B, working memory were tested. During VST, patients were asked to find 9 circles with digits written from 1 to 9 on a screen. Patients had to carefully watch the circles blink to press the circles in the order previously seen (for VST-F), or in backwards (for VST-B). With VSTs, visual attention and memory were tested. In VCPT, patients had to attentively watch the screen as numbers through 1 to 9 randomly showed one at a time. When a certain number ( 3 , in our case) appeared, the testee had to quickly press a button in front of them. For ACPT, the cue was delivered in sounds. Visual sustained attention was tested with VCPT. Auditory attention was tested with ACPT. TMT involved connecting randomly scattered numbers in order by touching the screen as fast and accurate as possible. The test also evaluated visual attention and cognitive processing. In WCST, which tested the executive function, cards were sorted in one out of three rules, which the patient had to deduce. RCPM tested visual intelligence by showing a series of pictures on the screen and acquiring patient to select the most appropriate picture for a missing space in a series of pictures. Subtests with simple enough instructions and as less verbal commands as possible were selected into the test. Tests were carried out in an equal environment for all subjects, by performing the tests in a quiet room created only for the evaluation.

Among various parameters used for each subtest, we selected a single specific score for every subtest for analysis. For DST and VST, the number of digits completed was taken. For ACPT and VCPT, the number of correct responses was selected. For TMT-A, the time (expressed 
as seconds) taken to complete the set was analyzed. For WCST, the number of categories completed by the patient was analyzed. For RCPM, the number of correct response was taken in to analysis.

The Korean version of Western Aphasia Battery (K-WAB) was used for language evaluation. Patients were evaluated by a single SLP for aphasia, and any type of aphasia determined by the SLP was included in group 3. As the measure of severity, aphasia quotient (AQ) of K-WAB was used and the scores of 4 domains (spontaneous speech, auditory comprehension, repetition and naming) were also collected.

The time interval between CNT and language evaluation was within one week.

\section{Statistical analysis}

To analyze the clinical characteristics among three groups, we performed ANOVA test. Shapiro-Wilk test was used to test normality of the distribution of CNT data samples of the three groups. Data failed to show normality for CNT scores, so we used Kruskal-Wallis test to investigate the differences in CNT scores of the three groups. For post-hoc analysis, Mann-Whitney test with Bonferroni correction was used. To look for any correla- tion between the nonlinguistic cognitive deficits and the degree of aphasia, Spearman correlation coefficient was calculated between each domain of K-WAB and the subtests of CNT. Statistical significance was conducted at $\mathrm{p}<0.05$ ( $\mathrm{p}<0.017$ for Mann-Whitney test with Bonferroni correction). Statistical analysis was conducted using SPSS ver. 16.0 (SPSS Inc., Chicago, IL, USA).

\section{RESULTS}

\section{Clinical characteristics}

Clinical characteristics of participants are summarized in Table 1. Mean age, educational years, and duration from onset were similar among all three groups, showing no statistically significant differences by ANOVA test.

Korean version of Mini-Mental Status Examination (K-MMSE) showed significantly lower scores in group 3 compared to other groups, which could be explainable by the presence of aphasia, and yet average showed a relatively high score of 19.6.

Various types of aphasia were present as the result of aphasia assessment. The types of aphasia in group 3 were mostly anomic and Broca's type. Mean K-WAB score in group 3 was 46.60 .

Table 1. Demographic and clinical characteristics

\begin{tabular}{|lccc}
\hline \multicolumn{1}{c}{ Characteristic } & Group 1 (n=36) & Group 2 (n=32) & Group 3 (n=26) \\
\hline Age (yr) & $59.2 \pm 12.3$ & $61.2 \pm 11.8$ & $54.7 \pm 12.8$ \\
\hline Gender (male:female) & $22: 14$ & $18: 14$ & $18: 8$ \\
\hline Education (yr) & $11.8 \pm 4.6$ & $9.6 \pm 3.0$ & $10.4 \pm 4.2$ \\
\hline Duration from stroke onset (day) & $32.8 \pm 16.4$ & $26.8 \pm 14.6$ & $28.0 \pm 14.5$ \\
Type of lesion (hemorrhagic:ischemic) & $13: 23$ & $11: 21$ & $15: 11$ \\
\hline K-MMSE & $25.1 \pm 3.5$ & $23.9 \pm 5.4$ & $19.6 \pm 6.8^{\mathrm{a})}$ \\
\hline Type of aphasia & & & \\
\hline Global & - & - & 2 \\
\hline Broca & - & - & 6 \\
Wernicke & - & - & 1 \\
\hline Anomic & - & - & 8 \\
\hline Transcortical sensory & - & - & 5 \\
\hline Conduction & - & - & 1 \\
\hline Unclassified & - & - & 2 \\
\hline K-WAB (Aphasia Quotient) & - & - & $46.60 \pm 20.82$ \\
\hline
\end{tabular}

Values presented as mean \pm standard deviation or number.

K-MMSE, Korean version of Mini-Mental Statue Examination; K-WAB, Korean version of Western Aphasia Battery.

${ }^{a)}$ Statistically significant $(\mathrm{p}<0.05)$ difference between group 3 and other groups. 


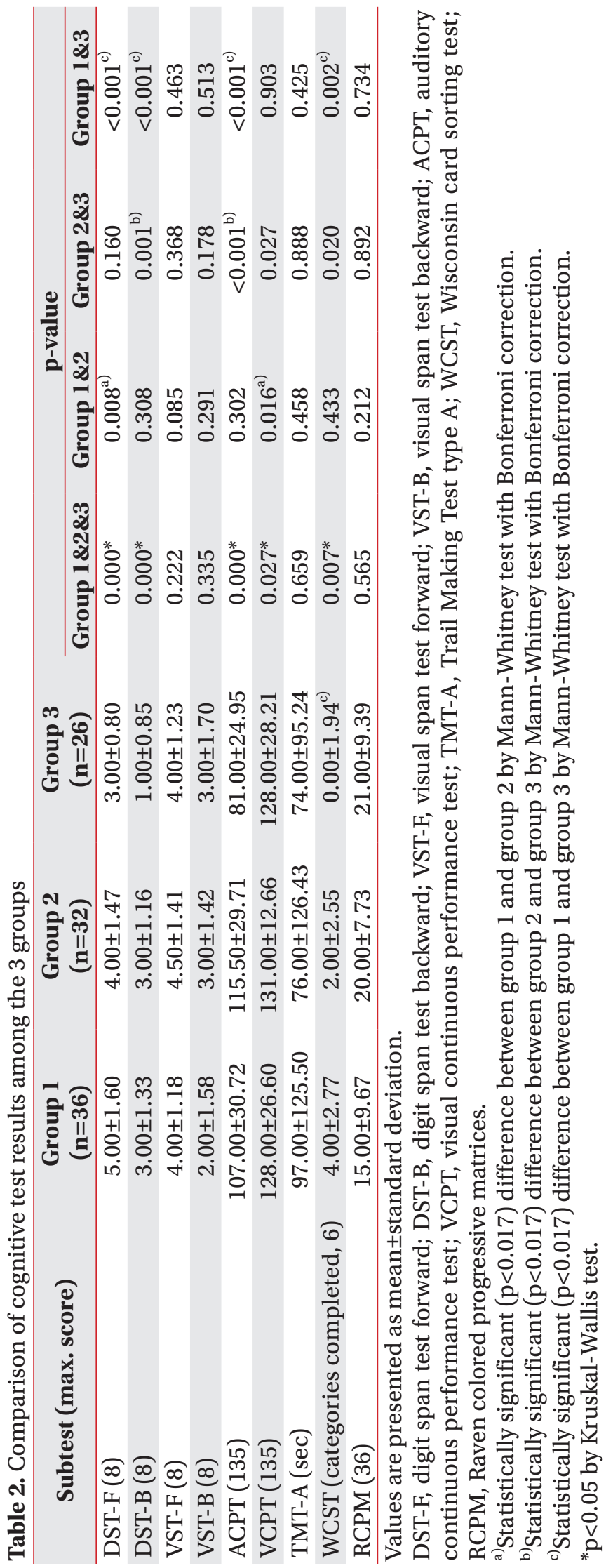

\section{Patterns of cognitive deficits in the three groups}

Results of Kruskal-Wallis test between groups 1, 2, and 3 are shown in Table 2. Between groups 1 and 2, DST-F showed significantly $(\mathrm{p}=0.014)$ higher score in group 1 and VCPT showed significantly $(\mathrm{p}<0.05)$ higher score in group 2 . Other tests did not show statistically significant difference between groups 1 and 2 . The DST-F $(\mathrm{p}<0.001)$ and WCST $(p=0.002)$ scores of patients in group 3 were significantly lower than in group 1 . Scores of VCPT and WCST in group 3 were significantly lower than group 2 with borderline statistical significance $(p=0.027$ and $\mathrm{p}=0.020$, respectively). In addition, test scores of DST-B ( $\mathrm{p}<0.001$ and $\mathrm{p}=0.001$, respectively) and ACPT $(\mathrm{p}<0.001$ and $p<0.001$, respectively) in group 3 were significantly lower than that in both groups 1 and 2. The scores of RCPM were similar to each other among the three groups.

Correlation between aphasia severity and cognitive tests Correlation coefficients calculated between AQ and scores of each test in group 3 are shown in Table 3 . The subtests that had statistically significant correlation with AQ were DST-F ( $\mathrm{p}=0.001)$, DST- $\mathrm{B}(\mathrm{p}=0.029)$, and TMT-A $(p=0.004)$. Other subtests failed to show any significant correlation with the aphasia severity.

Among the cognitive tests, DST-F showed significant correlation between all four domains of K-WAB, namely spontaneous speech, auditory comprehension, repetition, and naming. Auditory comprehension, among other domains, had the most statistically significant correlation with the cognition subtests: DST-F $(\mathrm{p}=0.010)$, DST-B ( $p=0.009)$, VST-F $(p=0.005)$, VST-B $(p=0.030)$ and TMT-A ( $\mathrm{p}=0.002)$. Other than comprehension, naming also showed significant correlation with DST-F ( $\mathrm{p}=0.010)$, DST-B ( $p=0.004)$ and TMT-A $(p=0.003)$.

\section{DISCUSSION}

One of the purposes of this study was to reveal the differences in cognitive deficits of right and left hemispheric stroke patients and furthermore to elucidate the characteristics in cognitive functions in aphasic patients. Our results showed no significant difference between right and left brain damaged patients without aphasia in most of the tests, but DST-F showed a significantly superior performance in the right hemispheric stroke patients, 
Table 3. Correlation coefficients between language domains consisting K-WAB and scores of cognitive tests in group 3

\begin{tabular}{lccccc}
\hline Subtest & Spontaneous speech & Comprehension & Repetition & Naming & AQ \\
\hline DST-F & $0.626^{\text {a) }}$ & $0.576^{\text {b) }}$ & $0.634^{\text {b) }}$ & $0.576^{\text {a) }}$ & $0.679^{\text {b) }}$ \\
\hline DST-B & 0.430 & $0.581^{\text {b) }}$ & 0.259 & $0.627^{\text {b) }}$ & $0.500^{\text {a) }}$ \\
\hline VST-F & 0.342 & $0.593^{\text {b) }}$ & 0.270 & 0.393 & 0.498 \\
\hline VSF-B & 0.295 & $0.473^{\text {a) }}$ & 0.022 & 0.384 & 0.349 \\
\hline ACPT & -0.107 & 0.431 & -0.169 & -0.012 & 0.062 \\
\hline VCPT & -0.067 & 0.389 & -0.233 & 0.008 & 0.085 \\
\hline TMT-A & -0.526 & $-0.706^{\text {b) }}$ & -0.395 & $-0.700^{\text {b) }}$ & $-0.674^{\text {b) }}$ \\
\hline WCST & -0.195 & 0.283 & -0.262 & 0.258 & -0.039 \\
\hline RCPM & 0.190 & 0.413 & 0.169 & 0.008 & 0.414 \\
\hline
\end{tabular}

K-WAB, Korean version of Western Aphasia Battery; AQ, aphasia quotient; DST-F, digit span test forward; DST-B, digit span test backward; VST-F, visual span test forward; VST-B, visual span test backward; ACPT, auditory continuous performance test; VCPT, visual continuous performance test; TMT-A, Trail Making Test type A; WCST, Wisconsin card sorting test; RCPM, Raven colored progressive matrices.

${ }^{a)}$ Statistically significant $(\mathrm{p}<0.05)$ correlation.

${ }^{\mathrm{b}}$ Statistically significant $(\mathrm{p}<0.01)$ correlation.

which has been already described in previous studies [12], and VCPT showed significantly higher score in the patients with left brain damage without aphasia. Traditionally accepted concept that visual memory is related to right brain lesions $[13,14]$ failed to be proved, but visual attention was significantly affected in the patients with right brain damage.

Our study was in accordance with an earlier study by Burgio and Basso [1] that reported that left hemisphere damaged patients performed significantly worse in verbal memory tasks. In the comparison between left hemisphere damaged aphasic and non-aphasic patients, working memory seemed to be significantly impaired in the aphasia group. Although performance of digit span test is known to be affected by language skills, given that there was no significant difference between the two groups in digit span forward test, we could conclude that the significantly low performance of DST-B in aphasia group can be accounted mostly to the differences in working memory ability between the two groups. It's well agreed that adults with aphasia present with working memory deficit that contributes to their language processing impairments [15]. A recent study by Kasselimis et al. [16] has shown that rather than the left hemisphere lesion, presence of aphasia itself is responsible for the differences in memory tasks, and our study also demonstrated that verbal working memory may be dependent on the presence of aphasia rather than the location of the lesion. Moreover, Tompkin et al. [17] used modified auditory span to evaluate working memory deficits and found that language comprehension was correlated with the performance. Recently, some studies have focused on attention deficits, one of the most frequent cognitive symptoms after stroke [18], with relations to aphasia, showing attention deficits present in most aphasic patients [12]. In this study, vigilance, assessed by visual and auditory CPT, also seemed to be impaired in aphasic patients compared to left-hemisphere damaged non-aphasic patients, although the impairment of visual sustained attention was only of borderline significance $(\mathrm{p}=0.027)$. As the correlation analysis showed no significance between AQ and ACPT or VCPT scores, the relative impairment of sustained attention compared to other stroke patients, in addition to language impairment, may be responsible for the differences in the CPT scores. However, it should be noted that, even though there was no significant correlation between AQ and ACPT score, there is still a possibility that the difference in ACPT performance may be due to difference in language perception skills.

Executive function was assessed by WCST in our study. Although the scores did not show significant differences between groups 2 and 3, there was a marginally significant differences $(p=0.020)$ between the 2 groups, with a clear difference between groups 1 and $3(\mathrm{p}=0.002)$. Meanwhile, in the correlation test with the aphasia severity, WCST did not show any correlation with AQ, which 
may infer that the degree of language impairment did not contribute to the WCST performance and the differences to some extent might reflect the differences in executive functions, although as WCST is a test of some verbal load, the language factor has to be considered.

And for nonverbal intelligence measured by RCPM, the three groups showed similar performances which is consistent with previous report [7]. The study by Kertesz and McCabe [7] stated that RCPM performance did not directly relate to the severity of aphasia, and that the visual intelligence is impaired in aphasics to a variable extent. In coherence with our study, Seniow et al. [9] showed that abstract thinking and visuo-spatial working memory were impaired in general in patients with post-stroke aphasia, but were heterogeneous with regard to their deficits.

In the correlation study between $\mathrm{AQ}$ and scores of cognitive tests, only DST-F, DST-B, and TMT-A scores showed statistical significance. Severity of aphasia was significantly correlated with verbal attention and verbal working memory. The significant correlation with DSTs is also likely due to the fact that DSTs also reflects the ability of how well one accepts auditory information. Other than DSTs and TMT, correlation coefficients were variable among the cognitive function tests without statistical significance. In the earlier study, Kertesz and McCabe [7] showed that visual analogic intelligence and the severity of aphasia had low correlation and that the performance of RCPM was comparable to the control group without language impairment. Fucetola et al. [19] also stated that language competence was only a partial factor in explaining the nonverbal cognitive performance. Our study also supports the notion that the severity of aphasia does not fully explain the nonverbal cognitive ability of aphasics, suggesting other possible factors and the dominant role of the right hemisphere in nonverbal cognition.

In our study, among other language components, comprehension had the most correlation with cognitive performance. Kertesz and McCabe [7] stated that comprehension was the most important 'language factor' limiting cognitive performance in aphasia. Fucetola et al. [19] also showed a similar finding that auditory comprehension rather than expressive component had correlation with cognition. In a previous study regarding nonlinguistic cognitive deficits in the recovery of aphasia, improvement of two language functions, naming and comprehension, was associated with visuo-spatial working memory [9].

Limitations to our study include its relatively small sample size compared to the number of variables involved. In addition, analysis on individual data of lesion site and size were not performed in this study. This study also lacks the control group of healthy subjects, making the analysis of cognitive performances less clear in patients with aphasia. Studies concerning lesion location in more detail may add some more insight to the understanding of cognitive impairment in left hemispheric patients with aphasia. Patterns of cognitive impairment at an individual level were not analyzed, making it unable to identify previously well reported heterogeneity in the types and severity of cognitive symptoms in aphasia patients [12]. Future study using larger sample size including healthy controls with prospective design is needed to investigate the clear relationship between cognitive function and aphasia. Also, follow-up measure may provide more insight to the role of cognitive function in aphasia recovery.

In summary, cognitive deficits can be accompanied in patients presenting with post-stroke aphasia, and there may be possible associations between language and cognitive measures. Therefore, detection and treatment towards coexisting cognitive impairment may be helpful or even necessary for efficient aphasia treatment and successful rehabilitation.

Our results accorded relatively well with prior aphasia research $[9,20,21]$ identifying some cognitive deficits in post-stroke aphasics.

\section{CONFLICT OF INTEREST}

No potential conflict of interest relevant to this article was reported.

\section{ACKNOWLEDGMENTS}

This work was supported by the National Research Foundation of Korea funded by the Korean Government (NRF-2013S1A3A2043454).

\section{REFERENCES}

1. Burgio F, Basso A. Memory and aphasia. Neuropsy- 
chologia 1997;35:759-66.

2. Beeson PM, Bayles KA, Rubens AB, Kaszniak AW. Memory impairment and executive control in individuals with stroke-induced aphasia. Brain Lang 1993;45:253-75.

3. Helm-Estabrooks N. Cognition and aphasia: a discussion and a study. J Commun Disord 2002;35:171-86.

4. Kauhanen ML, Korpelainen JT, Hiltunen P, Maatta R, Mononen H, Brusin E, et al. Aphasia, depression, and non-verbal cognitive impairment in ischaemic stroke. Cerebrovasc Dis 2000;10:455-61.

5. El Hachioui H, Visch-Brink EG, Lingsma HF, van de Sandt-Koenderman MW, Dippel DW, Koudstaal PJ, et al. Nonlinguistic cognitive impairment in poststroke aphasia: a prospective study. Neurorehabil Neural Repair 2014;28:273-81.

6. Postle BR. Working memory as an emergent property of the mind and brain. Neuroscience 2006;139:23-38.

7. Kertesz A, McCabe P. Intelligence and aphasia: performance of aphasics on Raven's coloured progressive matrices (RCPM). Brain Lang 1975;2:387-95.

8. Luriia AR. The working brain: an introduction to neuropsychology. London: Allen Lane; 1973.

9. Seniow J, Litwin M, Lesniak M. The relationship between non-linguistic cognitive deficits and language recovery in patients with aphasia. J Neurol Sci 2009;283:91-4.

10. Fillingham JK, Hodgson C, Sage K, Lambon Ralph MA. The application of errorless learning to aphasic disorders: a review of theory and practice. Neuropsychol Rehabil 2003;13:337-63.

11. Vallila-Rohter S, Kiran S. Non-linguistic learning and aphasia: evidence from a paired associate and feedback-based task. Neuropsychologia 2013;51:79-90.

12. Murray LL. Attention and other cognitive deficits in aphasia: presence and relation to language and com- munication measures. Am J Speech Lang Pathol 2012; 21:S51-64.

13. D'Esposito M, Postle BR. The dependence of span and delayed-response performance on prefrontal cortex. Neuropsychologia 1999;37:1303-15.

14. Kessels RP, van Zandvoort MJ, Postma A, Kappelle LJ, de Haan EH. The Corsi Block-Tapping Task: standardization and normative data. Appl Neuropsychol 2000;7:252-8.

15. Wright HH, Fergadiotis G. Conceptualizing and measuring working memory and its relationship to aphasia. Aphasiology 2012;26:258-78.

16. Kasselimis DS, Simos PG, Economou A, Peppas C, Evdokimidis I, Potagas C. Are memory deficits dependent on the presence of aphasia in left brain damaged patients? Neuropsychologia 2013;51:1773-6.

17. Tompkins CA, Bloise CG, Timko ML, Baumgaertner A. Working memory and inference revision in braindamaged and normally aging adults. J Speech Hear Res 1994;37:896-912.

18. Lesniak M, Bak T, Czepiel W, Seniow J, Członkowska A. Frequency and prognostic value of cognitive disorders in stroke patients. Dement Geriatr Cogn Disord 2008;26:356-63.

19. Fucetola R, Connor LT, Strube MJ, Corbetta M. Unravelling nonverbal cognitive performance in acquired aphasia. Aphasiology 2009;23:1418-26.

20. Kalbe E, Reinhold N, Brand M, Markowitsch HJ, Kessler J. A new test battery to assess aphasic disturbances and associated cognitive dysfunctions: German normative data on the aphasia check list. J Clin Exp Neuropsychol 2005;27:779-94.

21. Yeung O, Law SP. Executive functions and aphasia treatment outcomes: data from an ortho-phonological cueing therapy for anomia in Chinese. Int J Speech Lang Pathol 2010;12:529-44. 